\title{
An Android-Based Driver Drowsiness Monitoring System
}

\author{
Hu Jian-feng Mu Zhen-dong Wang Ping \\ Institute of Information Technology, Jiangxi University of Technology, Nanchang 330098, China
}

Keywords: Android; EEG; Driver; Drowsiness

\begin{abstract}
The traffic accident has become one of important factors of abnormal deaths, and the status of the driver is especially important for traffic accident. This paper describes an Android-based driver drowsiness monitoring system. The system collect EEG signal of driver, analysis EEG data and extract feature, determine the condition of drowsiness, if yes, then give alert.
\end{abstract}

\section{Introduction}

More and more traffic accidents due to driver's lowering alert level have become a serious social problem. These accidents are the results of the drivers' conditions. However, most of these accidents are related to drowsiness and driver fatigue. Driver fatigue related accidents would be more serious, leading to serious injury and death. About $30 \%$ of all the traffic accidents have been caused by drowsiness. Results show that the driving performance deterioration with increased drowsiness leading to accidents are more than $20 \%$ of all the vehicle accident. One can use many different techniques to analyze driver's drowsiness. Basic techniques, such as image processing based technique, Electroencephalograph (EEG) based technique, heart rate based technique, blood pressure based technique, temperature based technique, and eyelid closure based technique.

\section{System Design}

System comprises several modules: EEG acquisition module; data analysis module; feature extraction module; basic framework module; Android module and alert module. Figure 1 illustrates an overview of the system design for the proposed system. The phone receives EEG data and an extraction process is performed to extract meaningful features from the received data; these features then serve as input models to an inference network to analyze the driver's vigilance level. An alert system is triggered if the statistical results indicate that the driver's alertness is predicted to be lower.

In the driving process, EEG acquisition device constantly gather driver's brain waves, send to the phone via Bluetooth, the APP inside of the phone receives the EEG signals and preprocess, then extract features. The APP judge whether the driver is drowsiness. If yes, the APP will take an alert display.

Figure. 1 System design overview consists of several modules where smartphone device receives data from EEG sensors that placed on the head of driver. 


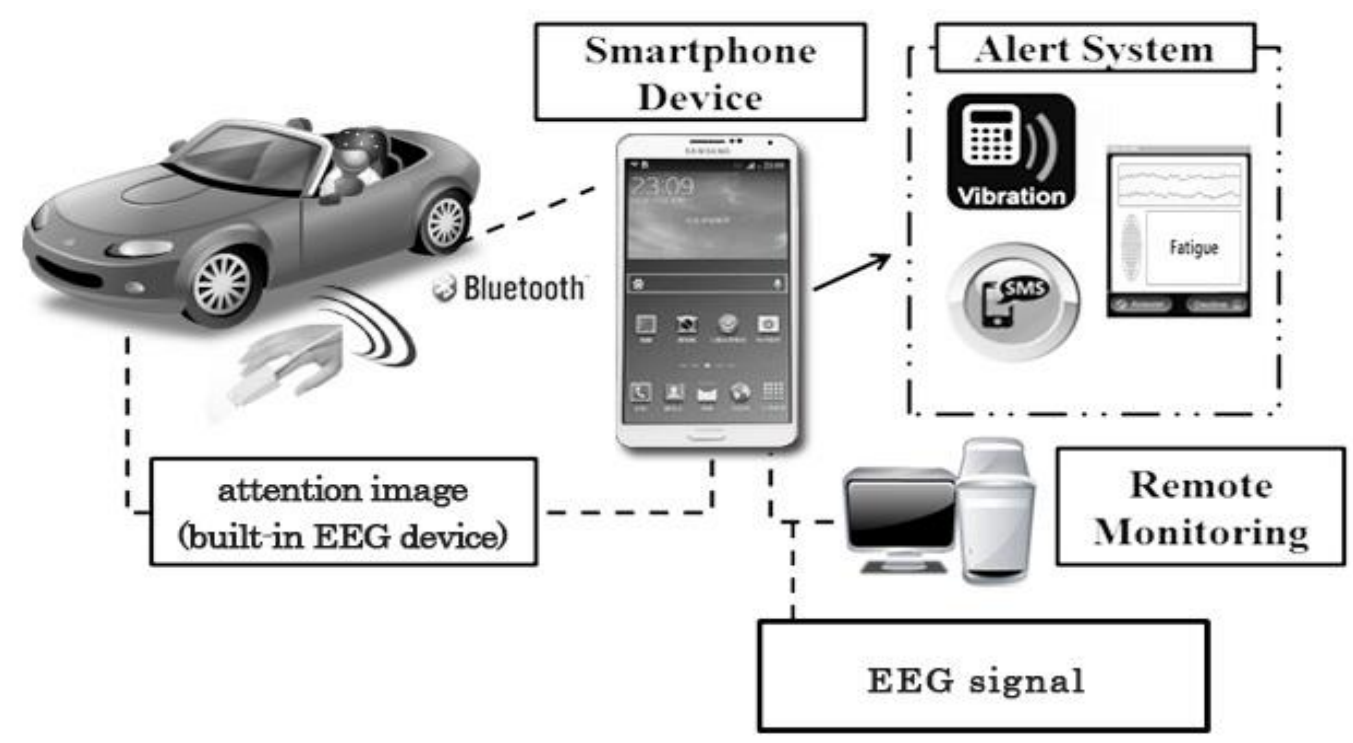

Figure. 1

Figure. 2 illustrates the flow chart for the application developed in a phone. Firstly, the application received the EEG data. Then, features are extracted.

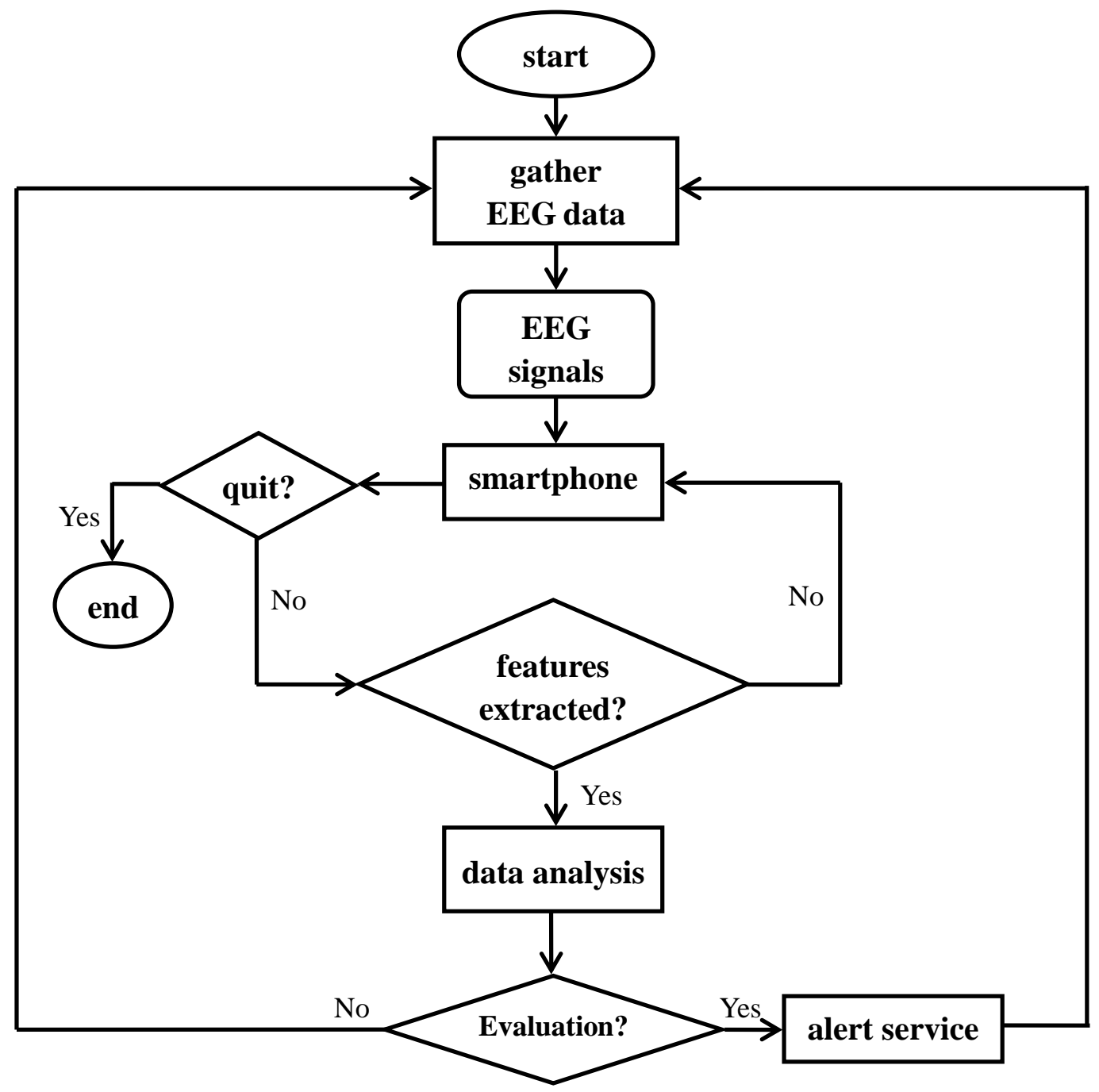

Figure. 2 Application flowchart. 
Wavelet analysis is a rapidly developing new collar domain in mathematics, which has both theoretical significance and profound and extensive application. The concept of wavelet transform is by French engineers engaged in J.Morlet oil signal processing is first proposed in 1974, by physical intuition and signal processing of the actual need to experience established inversion formula, it and Fourier transform, window Fourier transform (Gabor transform), this is a time and frequency domain transform, and so can effectively extract information from signal, multiscale analysis of functions or signals through extension and translation operations (Multiscale Analysis), solves many difficult problems cannot be solved by Fourier transform, and wavelet transform is known as "mathematical microscope", it is the milepost type harmonic analysis in the history of the development of. The properties of time is constant, the ideal tool for processing is still $\mathrm{Fu}$ Liye analysis. But the vast majority of signal in practical application is not stable, and is especially suitable for non-stationary signal is wavelet analysis tools.

Signal continuous wavelet transform for $f(t)$ :

$$
W T(a, b)=\frac{1}{\sqrt{a}} \int_{-\infty}^{\infty} \varphi\left(\frac{t-b}{a}\right) d t, a \neq 0
$$

Where $\varphi(t)$ is the mother wavelet, a is a scaling factor, B is the translation factor, if $\mathrm{a}=2$, the translation factor $\mathrm{b}=2 \mathrm{k}$; then the discrete wavelet transform into two formula:

$$
W T(a, b)=\int 2^{-i / 2} f(t) \varphi\left(2^{-i} t-k\right) d t, j, k \in Z
$$

\section{Results and Discussion}

In the process of fatigue prediction, a safety monitoring system must be able to notify a driver within a very short time period when a dangerous situation arises. Figure 3 demonstrates a few screenshots of the prototype system, which was developed on the Android platform. Figure 3 shows the main screen of the system that performs fatigue analysis in real-time.

Driving fatigue is one of the main reasons the accident at present, preventing the occurrence of driving fatigue can effectively reduce automobile accidents. However, due to technical and financial aspects, devices which prevent fatigue driving are not universal. Due to the rapid development and popularization of intelligent mobile terminal, a fatigue driving alert system of automobile based on brain wave is possible. And with the special equipment by installing the car driver fatigue warning system in intelligent mobile terminal, fatigue alert function can be realized, which can provide further guarantee for safe driving.

In the brain wave monitoring function, through the analysis of the EEG features, the software is unlimited by car model. At the same time, the system can also be combined with acceleration data, the engine running state and the vehicle traveling time, comprehensive data processing and analyzing whether the driver is fatigue driving state. 


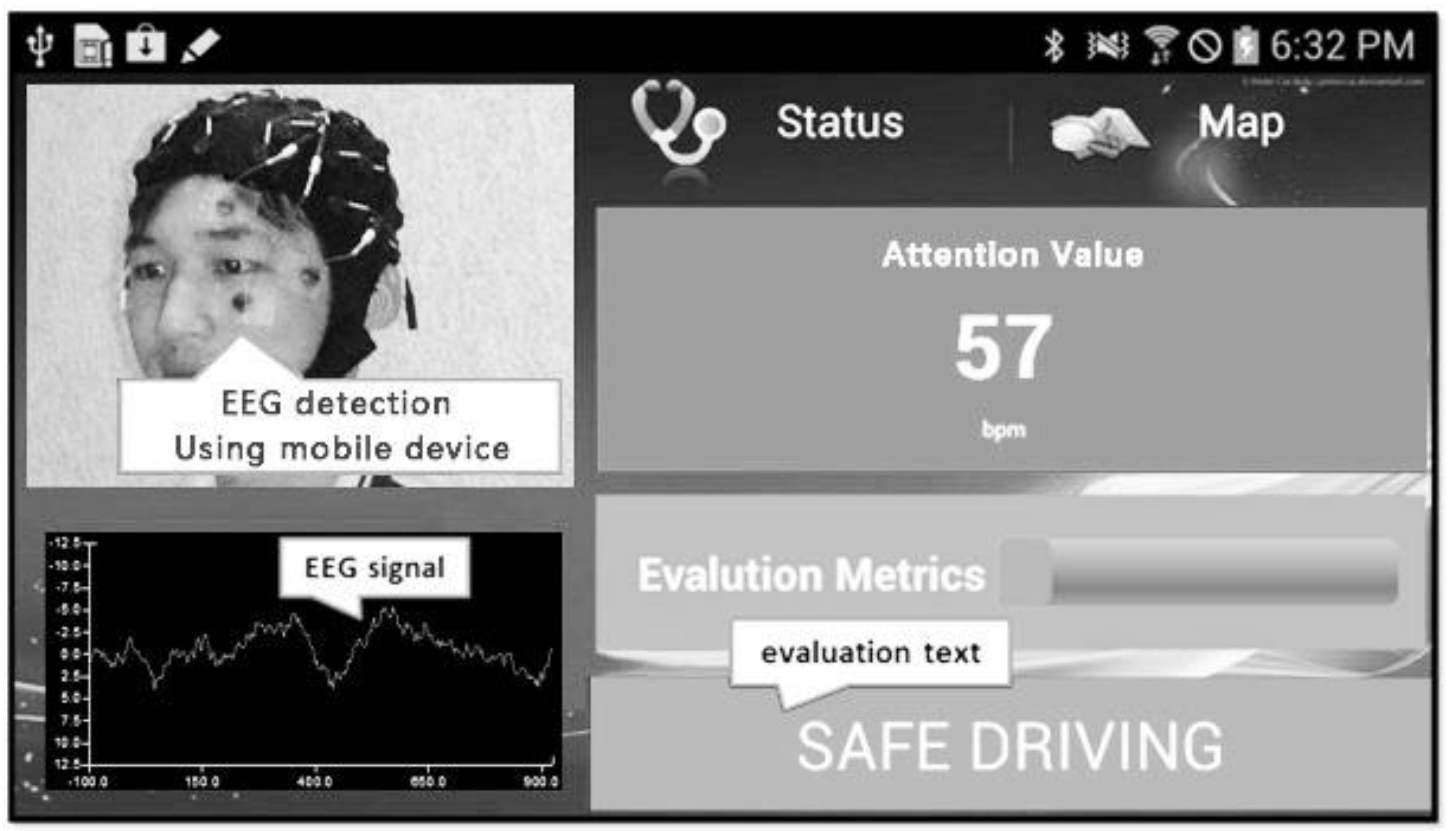

Figure. 3 Screenshots captured in a real Android-based phone.

\section{Conclusion}

This system uses drowsiness detection systems based on EEG to check drivers' condition. EEG based techniques are efficient but practically it is not suitable for driver to wear electrodes. This technique is much simpler and user friendly. There is a lot of scope in drowsiness detection using EEG.

\section{Acknowledgment}

This work was supported by Natural Science Foundation of Jiangxi Province [No 20142BAB207008] and project of Science and Technology Department of Jiangxi Province [No 2013BBE50051]. The authors thank Wang Ping for technique support.

\section{References}

[1] Hu JF, Mu ZD. Smart Car Security System by Using Biometrics Based on EEG [J]. $2013^{\text {2nd }}$ International Conference on Frontiers of Energy and Environment Engineering, ICFEEE 2013: 1047-1049.

[2] Lee BG, Chung WY. A Smartphone-Based Driver Safety Monitoring System Using Data Fusion [J]. Sensors, 2012, 17536-17552.

[3] Saini V, Saini R. Driver Drowsiness Detection System and Techniques: A Review [J]. International Journal of Computer Science and Information Technologies, 2014, 4245-4249.

[4] Vasavi D, Krishnaiah RV. Online detection of drowsiness using brain and visual information [J]. International Journal of Instrumentation Electrical Electronics Engineering, 2013, 31-34.

[5] Fuletra JD, Bosamiya D. A Survey on Driver's Drowsiness Detection Techniques [J]. International Journal on Recent and Innovation Trends in Computing and Communication, 2013, 816-819. 